OPEN ACCESS

Edited by:

Manuela Simoni,

University of Modena and Reggio

Emilia, Italy

Reviewed by:

Carlo Alviggi,

University of Naples Federico II, Italy

Sandro C. Esteves,

Androfert, Andrology and Human

Reproduction Clinic, Brazil

${ }^{*}$ Correspondence:

Bruno Lunenfeld

blunenfeld@gmail.com

Specialty section: This article was submitted to Reproduction,

a section of the journa

Frontiers in Endocrinology

Received: 26 April 2019

Accepted: 14 June 2019

Published: 03 July 2019

Citation:

Lunenfeld B, Bilger W, Longobardi S, Alam V, D'Hooghe T and Sunkara SK

(2019) The Development of Gonadotropins for Clinical Use in the

Treatment of Infertility.

Front. Endocrinol. 10:429. doi: 10.3389/fendo.2019.00429

\section{The Development of Gonadotropins for Clinical Use in the Treatment of Infertility}

\author{
Bruno Lunenfeld ${ }^{1 *}$, Wilma Bilger ${ }^{2}$, Salvatore Longobardi ${ }^{3}$, Veronica Alam ${ }^{4,5}$, \\ Thomas D'Hooghe ${ }^{3,6,7}$ and Sesh K. Sunkara ${ }^{8}$
}

${ }^{1}$ Faculty of Life Sciences, Bar-llan University, Ramat Gan, Israel, ${ }^{2}$ Medical Affairs Fertility, Endocrinology and General Medicine, Merck Serono GmbH, Darmstadt, Germany, ${ }^{3}$ Global Medical Affairs Fertility, Merck Healthcare KGaA, Darmstadt, Germany, ${ }^{4}$ Global Clinical Development, EMD Serono, Rockland, MA, United States, ${ }^{5}$ A Business of Merck KGaA, Darmstadt, Germany, ${ }^{6}$ Organ Systems, Group Biomedical Sciences, Department of Development and Regeneration, KU Leuven (University of Leuven), Leuven, Belgium, ${ }^{7}$ Department of Obstetrics and Gynecology, Yale University, New Haven, CT, United States, ${ }^{8}$ Assisted Conception Unit, King's College London, Guy's Hospital, London, United Kingdom

The first commercially available gonadotropin product was a human chorionic gonadotropin (hCG) extract, followed by animal pituitary gonadotropin extracts. These extracts were effective, leading to the introduction of the two-step protocol, which involved ovarian stimulation using animal gonadotropins followed by ovulation triggering using hCG. However, ovarian response to animal gonadotropins was maintained for only a short period of time due to immune recognition. This prompted the development of human pituitary gonadotropins; however, supply problems, the risk for Creutzfeld-Jakob disease, and the advent of recombinant technology eventually led to the withdrawal of human pituitary gonadotropin from the market. Urinary human menopausal gonadotropin (hMG) preparations were also produced, with subsequent improvements in purification techniques enabling development of products with standardized proportions of follicle-stimulating hormone (FSH) and luteinizing hormone (LH) activity. In 1962 the first reported pregnancy following ovulation stimulation with $\mathrm{hMG}$ and ovulation induction with hCG was described, and this product was later established as part of the standard protocol for ART. Improvements in immunopurification techniques enabled the removal of LH from hMG preparations; however, unidentified urinary protein contaminants remained a problem. Subsequently, monoclonal FSH antibodies were used to produce a highly purified FSH preparation containing $<0.1 \mathrm{IU}$ of $\mathrm{LH}$ activity and $<5 \%$ unidentified urinary proteins, enabling the formulation of smaller injection volumes that could be administered subcutaneously rather than intramuscularly. Ongoing issues with gonadotropins derived from urine donations, including batch-to-batch variability and a finite donor supply, were overcome by the development of recombinant gonadotropin products. The first recombinant human FSH molecules received marketing approvals in 1995 (follitropin alfa) and 1996 (follitropin beta). These had superior purity and a more homogenous glycosylation pattern compared with urinary or pituitary FSH. Subsequently recombinant 
versions of LH and hCG have been developed, and biosimilar versions of follitropin alfa have received marketing authorization. More recent developments include a recombinant FSH produced using a human cell line, and a long-acting FSH preparation. These state of the art products are administered subcutaneously via pen injection devices.

Keywords: recombinant gonadotropin, follicle stimulating hormone, luteinizing hormone, fertility, pregnancy, pre-clinical, clinical

\section{INTRODUCTION}

It was observed in 1927, by Ascheim and Zondek, that the blood and urine of pregnant women contained a gonad-stimulating substance, human chorionic gonadotropin (hCG) $(1,2)$. SeegarJones and colleagues demonstrated in the 1940s that hCG was produced by the placenta (3). In 1929, Zondek proposed, based on his experiments and those of Smith, that two hormones were produced by the pituitary gland, both of which stimulated the gonads (4-6). These hormones were described as gonadotropins and subsequently named follicle-stimulating hormone (FSH) and luteinizing hormone (LH), according to their specific actions. The biological activity of gonadotropins suggested that they might be useful for the treatment of patients who were infertile. These observations eventually led to the development of pure gonadotropin products that have enabled the birth of millions of children to people affected by infertility.

This review provides an overview of the major milestones in the development of gonadotropin products (Figure 1), as well as issues that may have affected decision making during the development processes, and summarizes the available evidence supporting the use of recombinant gonadotropin products for the treatment of infertility.

\section{HUMAN CHORIONIC GONADOTROPIN}

The first commercially available gonadotropin was an hCG extract launched by Organon in 1931 (4). However, the original product was of limited use owing to a lack of reproducibility, in part due to the use of animal units (mouse or rat) to

\footnotetext{
Abbreviations: AMH, anti-Müllerian hormone; ART, assisted reproductive technologies; BMI, body mass index; $\mathrm{CHO}$, Chinese hamster ovary; CI, confidence interval; CLBR, cumulative live birth rate; COS, controlled ovarian stimulation; CPR, clinical pregnancy rate; CTP, carbonyl-terminal peptide; EMA, European Medicine Agency; ESHRE, European Society of Human Reproduction and Embryology; EU, European Union; Fc, Fragment crystallisable; FSH, follicle-stimulating hormone; GnRH, gonadotropin releasing hormone; hCG, human chorionic gonadotropin; HEK, human embryonic kidney; hMG, human menopausal gonadotropin; HPLC, high performance liquid chromatography; ICSH, interstitial cell stimulating hormone; ICSI, intracytoplasmic sperm injection; IgG, immunoglobulin G; IRP, international reference product; IU, international units; IVF, in vitro fertilization; LBR, live birth rate; LH, luteinizing hormone; OHSS, ovarian hyperstimulation syndrome; OPR, ongoing pregnancy rate; OR, odds ratio; PCOS, polycystic ovary syndrome; $\mathrm{PD}$, pharmacodynamics; PK, pharmacokinetics; POR, poor ovarian response; RCT, randomized controlled trial; r-hCG, recombinant human chorionic gonadotropin; r-hFSH, recombinant human follicle-stimulating hormone; r-hLH, recombinant human luteinizing hormone; $\mathrm{RR}$, risk ratio; u-FSH, urinary follicle-stimulating hormone; u-hCG, urinary human chorionic gonadotropin; USA, United States of America; WHO, World Health Organization.
}

measure bioactivity (7). Reproducibility was greatly improved in 1939 when the League of Nations developed the international standard for hCG; one International Unit (IU) of hCG was defined as the activity contained in $0.1 \mathrm{mg}$ of the reference hCG preparation which was pooled from six sources (8). Following the introduction of this standard, purified hCG preparations extracted from the urine of women during the first half of pregnancy, with bioactivity up to $8,500 \mathrm{IU} / \mathrm{mL}$, became available $(9,10)$.

\section{Clinical Use}

In women, hCG is used during infertility treatment to trigger final follicular maturation and ovulation, as well as for luteal phase support. In men, it is used to stimulate production of testosterone by the Leydig cells in cases of hormone deficiency as well as in male hypogonadism.

\section{ANIMAL PITUITARY GONADOTROPINS}

The first animal pituitary gonadotropin was swine pituitary gonadotropin [containing both FSH and LH $(11,12)$ ], followed by hog and sheep pituitary extracts and pregnant mare serum gonadotropin $(2,4,7,13)$. With the availability of both placental and pituitary hormones, the two-step protocol for ovarian stimulation using an animal gonadotropin followed by final maturation and triggering with hCG, was introduced for women in 1941 by Mazer and Ravetz $(2,14)$. However, owing to their non-human origin, the ovarian response to animal gonadotropins was only maintained in women for a limited duration because of human-animal immune recognition $(2,15)$.

As a result of the limited clinical value of the animal gonadotropins, human pituitary gonadotropins extracted either post-mortem from human pituitaries or from the urine of postmenopausal women were investigated (2).

\section{CADAVERIC HUMAN PITUITARY GONADOTROPINS}

In 1958, Gemzell extracted FSH from pituitaries obtained from human cadavers and reported successful follicle development using this preparation, which was later given to women together with hCG to induce ovulation $(16,17)$. In 1963, ovarian stimulation with cadaveric human pituitary gonadotropin in hypophysectomised individuals was successfully performed by Bettendorf et al. (7). Owing to their source, these products were produced by several government agencies. Although used successfully for a number of years, these human pituitary 


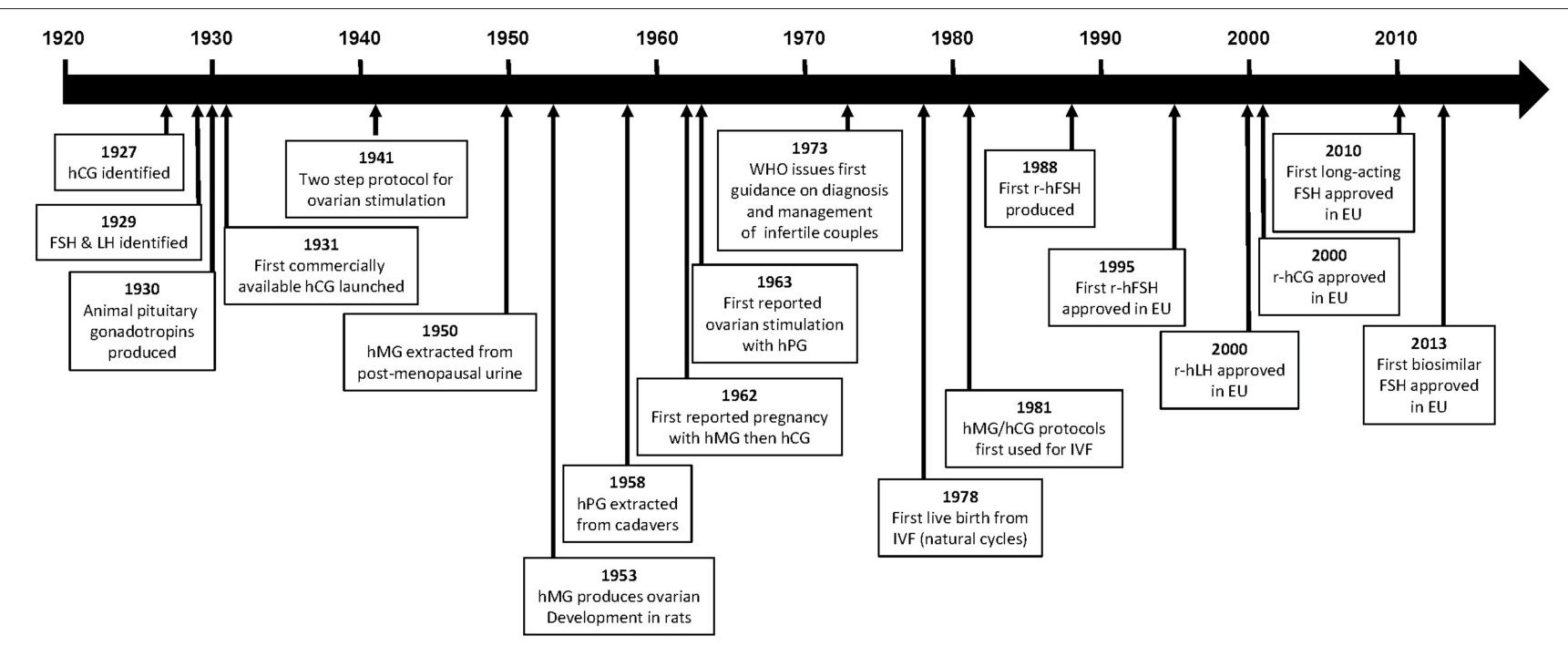

FIGURE 1 | Time line of major events in the development of gonadotropins. CHO, Chinese hamster ovary; FSH, follicle-stimulating hormone; hCG, human chorionic gonadotropin; hMG, human menopausal gonadotropin; LH, luteinizing hormone; r-hCG, recombinant human chorionic gonadotropin; r-hFSH, recombinant human follicle-stimulating hormone; $r-h L H$, recombinant human luteinizing gonadotropin.

preparations were discontinued in the 1980s because of supply problems and the risk for Creutzfeldt-Jakob disease that resulted from the source of these products (human cadavers) $(2,4,18-20)$.

\section{HUMAN MENOPAUSAL GONADOTROPIN}

Human menopausal gonadotropin (hMG), which contains two gonadotropin components corresponding to the pituitaryrelated hormones, FSH and LH, was first successfully extracted from the urine of post-menopausal women in 1950. In 1953 hMG was shown to produce ovarian stimulation in female hypophysectomised infantile rats, and Leydig cell stimulation and full spermatogenesis in male hypophysectomised infantile rats $(2,4,21)$. These experiments suggested that hMG would be useful in humans; however, to enable clinical testing, large-scale extraction and purification methods were required, in addition to an agreed standard to enable reproducibility. Furthermore, the starting dose for humans needed to be established. The first hMG preparations were registered by Serono in Italy in 1950, but these were impure in terms of protein content and did not have standardized proportions of FSH and LH. Subsequent preparations contained equal proportions of FSH and LH (for example, Pergonal 75 contained 75 IU FSH and 75 IU LH), in addition to other unwanted urinary proteins $(2,4)$. Additionally, the bioactivity of the first hMG preparations was measured in "animal" units (mouse or rat); a "rat unit" was the minimum amount of preparation required to induce oestrus in 28-day old female rats ("mouse units" were defined in a similar manner). The bioactivity, therefore, varied depending on the strain of animal used and a uniform standard was required to facilitate clinical use.

The first reference standard for hMG was based upon batches produced by kaolin extraction of menopausal urine (hMG 20, hMG 20a, and hMG 24) and provided by Organon Newhouse
$(2,7)$. However, by 1959 , most of the reference product had been used and further batches could not be provided. At this time, the Serono Institute in Rome offered $50 \mathrm{~g}$ of Pergonal 23 (containing equal proportions of FSH and $\mathrm{LH}$ ) to act as the reference preparation and this material was subsequently used as the International Reference Preparation (IRP) $(2,7,22)$. As well as facilitating greater reproducibility in clinical testing, the study of day-to-day variation of gonadotropins and steroid secretion during the normal menstrual cycle and during pregnancy was enabled by the availability of a reference product $(2,4,7,23)$. The aim of these studies was to understand the fundamental variability of gonadotropins in women so that these physiological concepts or patterns could be applied in future clinical tests.

Clinical trials were initiated and, in 1959, hMG (150 U/d for 4 days) was demonstrated to induce the expected, desirable changes in the endometrium and vaginal epithelium (24) and to induce steroid secretion, in women with anovulatory, hypogonadotropic hypopituitary and primary amenorrhea (2, 4, 22, 24-26). This was followed 3 years later by a report from Lunenfeld et al. of the first pregnancy in a patient with hypopituitary hypogonadotropic amenorrhea following ovulation induction with hMG and final oocyte maturation with hCG, no adverse events were reported for this pregnancy (26). This approach subsequently became the standard protocol for ovulation induction treatment of infertility $(2,26,27)$.

The World Health Organization (WHO) Expert Committee of Biological Standardization defined, in 1972, the IU for both FSH and LH (then named interstitial cell stimulating hormone [ICSH]) as the respective activities contained in $0.2295 \mathrm{mg}$ of the IRP of hMG (28). The use of IU depends upon determination of the linearity of the bioactivity of the gonadotropin product. The bioactivity of FSH, for example, is determined by the Steelman-Pohley bioassay. This bioassay is based on comparison between the test FSH preparation and the international reference 
standard (defined by the WHO) of FSH-induced augmentation of ovarian weight in immature female rats co-treated with a high dose of hCG (29). One year later, in 1973, the WHO issued their first guidance on the diagnosis and management of infertile couples, recommending an effective daily dose of 150225 IU hMG for hypogonadotropic patients (WHO Group I), and 75-150 IU for anovulatory normogonadotropic patients (WHO Group II) $(24,26,30)$.

Steptoe and Edwards pioneered in vitro fertilization (IVF) procedures using natural cycles, achieving the first live birth in 1978 (31); one pregnancy was reported following 101 attempts (32). However, in 1981, Jones and Jones established hMG/hCG protocols as described by Lunenfeld et al. (26) as the standard approach for ovarian stimulation in assisted reproductive technologies (ART), achieving one pregnancy after three attempts $(33,34)$. These protocols were later revised when the outcome of ovarian stimulation in ART treatment changed from mono-follicular to multi-follicular development (4).

Improvements in purification techniques enabled the development of an hMG preparation with fewer impurities. However, these extraction steps also removed LH activity (22) and hCG had to be added to re-establish the FSH:LH ratio, resulting in highly purified hMG containing approximately $30 \%$ identified impurities that varied from batch to batch (2). Polyclonal immunopurification techniques also resulted in an FSH preparation devoid of LH activity (35). However, this preparation still contained many unwanted urinary proteins. The development of monoclonal FSH antibodies to replace the polyclonal antibodies allowed greater purification of urinary products resulting in a highly purified FSH preparation (Metrodin HP [EU]; Fertinex [USA]; highly purified urofollitropin) containing about 9000 IU of FSH per mg of protein, $<0.1$ IU LH activity and $<5 \%$ unidentified urinary proteins. This enabled the formulation of smaller injection volumes and subcutaneous, rather than intramuscular, administration (2). The currently available hMG preparations are considered safe with the most common adverse events, as reported by clinical trials, being ovarian hyperstimulation, abdominal pain, headache, enlarged abdomen, inflammation at the injection site, pain at the injection site and nausea; the incidence rate of these events was 2-7\% (36).

Despite these advances in the preparation of urinary gonadotropin products, supplies were limited owing to the finite donor supply, and batch-to-batch variability was an issue because of the source (2). These issues were overcome by the development of recombinant human FSH (r-hFSH) and subsequently recombinant human LH (r-hLH) and hCG (rhCG) (2).

\section{Clinical Use}

hMG is approved for development of a single Graafian follicle in women with anovulation and multifollicular development in women undergoing controlled ovarian hyperstimulation as part of ART treatment. hMG has also been demonstrated to be effective for the induction or restoration of secondary sexual development and fertility due to androgen deficiency in males with hypogonadotropic hypogonadism, when used in combination with hCG (37-39).

\section{RECOMBINANT GONADOTROPINS}

Recombinant biological products are proteins produced using recombinant DNA technology that utilize biological processes to produce large molecule drugs that cannot be produced using synthetic chemistry. Recombinant gonadotropins were developed to avoid the limitations inherent to the earlier urinederived gonadotropin products, since recombinant products can be produced in large volumes with high purity and without variability in composition. As with $\mathrm{hMG}$, the recombinant products can be used for the treatment of both male and female infertility.

\section{Follicle-Stimulating Hormone}

There are currently three r-hFSH products on the market: follitropin alfa, follitropin beta, and follitropin delta. A fourth product, follitropin epsilon, has been reported as being in development (40). Follitropin alfa and follitropin beta are produced in Chinese hamster ovary $(\mathrm{CHO})$ cell lines, whereas follitropin delta is produced in human fetal retinal cells; all these r-hFSH products have an amino acid sequence identical to that of endogenous human FSH. FSH has a relatively short biological half-life of about 1 day (40), necessitating daily administration. There has therefore been interest in long-acting formulations, and one such product is available, the long-acting r-hFSH analog corifollitropin alfa [elimination half-life: corifollitropin alfa, 70 (59-82) hours (41); follitropin alfa, terminal elimination half-life $24 \mathrm{~h}(42)]$.

\section{Follitropin Alfa and Follitropin Beta}

The originator follitropin alfa (GONAL-f; Merck KGaA, Darmstadt, Germany) was first produced by Serono (a predecessor company of Merck KGaA) in 1988 and received a marketing license for clinical use in both women and men in the EU in 1995 (40) and in the USA in 2004 (42). Subsequently, two biosimilar versions of follitropin alfa have become available, for use in both women and men, Ovaleap (Teva B.V., Haarlem, the Netherlands), which received marketing authorization in Europe in 2013 (43), and Bemfola (Afolia, Finox Biotech AG, Balzers, Liechtenstein), which received marketing authorization in Europe in 2014 (44). The biosimilars are not currently approved in the USA. The safety profile of the biosimilar follitropin alfa preparations is similar to that of the originator product $(43,44)$. Follitropin beta (Puregon; Merck \& Co., Kenilworth, NJ) received marketing authorization in Europe in 1996 and in the USA (Follistim AQ) in $2004(45,46)$. The risk/benefit balance of follitropins alfa and beta are considered positive, with the main adverse events reported being headache, ovarian cysts, local injection site reactions (e.g., pain, erythema, hematoma, swelling, and/or irritation at the site of injection) and mild or moderate OHSS (40, 43, 44).

Although both follitropin alfa and follitropin beta are produced in $\mathrm{CHO}$ cells, the vectors used for gene expression differ. Follitropin alfa is produced in $\mathrm{CHO}$ cell lines that 
have been transfected with separate expression vectors for the $\alpha$ - and $\beta$-FSH genes, the master cell cultures having been selected to co-amplify both genes (47), whereas follitropin beta (Puregon) is produced in $\mathrm{CHO}$ cell lines transfected with a single expression vector containing both $\alpha$ - and $\beta$-FSH genes (48). Culture processes also differ, in that large-scale culture of follitropin alfa occurs in a bioreactor, followed by purification of the culture supernatant by an ultrafiltration step and five chromatographic steps, with the main chromatographic purification step achieved through immunoaffinity (using a murine-derived anti-FSH monoclonal antibody) (47). Following large-scale culture of follitropin beta, r-hFSH is isolated from culture supernatant by a series of chromatographic steps including anion and cation exchange chromatography, hydrophobic interaction chromatography and size exclusion chromatography (48).

Owing to differences in the production and purification of follitropin alfa and follitropin beta there are differences in their glycosylation, and they have different sialic acid residue compositions and isoelectric coefficients. The isoelectric point band (pI) for follitropin alfa is narrower than that of follitropin beta (4-5 and 3.5-5.5, respectively), furthermore, follitropin alfa contains fewer isoforms with a pI $<4$ ( 9 and $<24 \%$, respectively) (49). These variations result in follitropin alfa being slightly more acidic and follitropin beta more basic, which influences their metabolic clearance, half-life (Table 1), and biological activity $(48,49,54)$. The variance in mean specific FSH activity between follitropin alfa and follitropin beta (13,645 and 7,000$10,000 \mathrm{U} / \mathrm{mg}$ protein, respectively) affects the amount of protein required per injection (55). Follitropin alfa was originally dosed in IU based on its bioactivity in the Steelman-Pohley assay. However, owing to the consistency of the preparation it was possible to determine its specific activity, which is the ratio of the bioactivity (IU) to the protein content (mg, determined by size exclusion HPLC). Follitropin alfa can therefore be provided in injection devices filled-by-mass, which resulted in more consist ovarian response and reduced cycle cancelation rate, intra-cycle dose adjustment and repetitive monitoring $(56,57)$.

Despite the disparities between follitropin alfa and follitropin beta, results of head-to-head clinical studies and retrospective studies comparing the two products for ovarian stimulation in women undergoing IVF have shown no significant differences between the preparations in terms of efficacy or safety (58-61). In the largest randomized prospective comparison, conducted in 172 women treated with follitropin alfa and 172 women treated with follitropin beta, a dose of $150 \mathrm{IU} /$ day resulted in 13.0 and 12.4 oocytes obtained with each treatment (primary outcome), respectively, whereas at a dose of 300 IU/day, numbers were 6.1 and 7.1, respectively (60). Clinical pregnancy rates (secondary outcome) were similar with both preparations; $33.5 \%$ per cycle and $37.4 \%$ per embryo transfer with follitropin alfa 150 IU/day and $32.9 \%$ per cycle and $36.4 \%$ per embryo transfer with follitropin beta $150 \mathrm{IU} /$ day (60).

The two biosimilar follitropin alfa products (Ovaleap and Bemfola) are considered to be similar to the reference product, GONAL-f; however, as a result of post-translational modifications, their structures are not identical. This is the result of differences in the processes used for their production and purification, including the cell line (despite all being produced using $\mathrm{CHO}$ cells) $(43,44)$. Specifically, differences in glycosylation were observed between the biosimilars and GONAL-f, with Bemfola showing higher antennarity, higher sialylation and higher batch-to-batch variability in activity compared with GONAL-f (62), whereas Ovaleap has a higher amount of the sialic acid N-glycolyl neuraminic acid compared with GONAL-f (63). For both biosimilars, the differences compared with GONAL-f were considered by regulatory agencies as minor and acceptable. Furthermore, a recent report on validation procedures for the Ovaleap manufacturing process showed the processes to be both robust and consistent, and that the resulting r-hFSH had similar characteristics to GONAL-f when molecular mass, primary structure, secondary structure, biological activity and product-related impurities were considered (64). Nevertheless, the observed differences may have a biological impact, including on FSH receptor activation, which has generated discussion regarding the potential clinical impact of these differences, particularly in "non-ideal" patients (i.e., older, poor or suboptimal responders or with worse prognosis factors), as by their nature there is always variation in biologics (65).

EMA guidelines recommend that, to determine clinical comparability, the efficacy of the reference and the similar biologic should be assessed in a randomized, parallel-group clinical trial, with number of oocytes retrieved as the primary endpoint, and ovarian hyperstimulation syndrome (OHSS) as an adverse reaction of special interest (66). Both Bemfola and Ovaleap have demonstrated equivalence to GONAL-f in terms of the number of oocytes retrieved (primary outcome) in women receiving $\operatorname{ART}(67,68)$. Other outcomes (secondary outcomes), including pregnancy and live birth rate (LBR), have been reported as comparable to, or not statistically significantly different from, the originator product (GONAL-f) $(69,70)$.

A post-hoc pooled analysis of data obtained from randomized controlled trials (RCTs) indicated that treatment with GONAL-f is associated with a higher $\operatorname{LBR}(p=0.037$; primary endpoint) and lower OHSS ( $p=0.011$; secondary endpoint) than treatment with the biosimilars (Bemfola or Ovaleap) (69). However, further meta-analysis of data obtained from RCTs, ongoing post marketing real-world data studies and pharmacovigilance data concerning the use of these biosimilars are needed to ensure comparable clinical efficacy of these therapies to the originator in clinical practice.

\section{Follitropin Delta}

Follitropin delta (Rekovelle; Ferring Pharmaceuticals, St. Prex, Switzerland) is produced using a human cell line, PER.C6 human fetal retinal cells, and received a marketing license in Europe in 2016 (51). It has a different glycosylation pattern from both follitropin alfa and follitropin beta (71). Follitropin delta has a higher proportion of tri- and tetra-sialylated glycans than follitropin alfa and also has both $\alpha 2,3$ - and $\alpha 2,6$-linked sialic acid whereas follitropin alfa only has $\alpha 2,3$-linked sialic acid (72). In vitro, follitropin delta was observed to be equivalent to follitropin alfa in a cell-free FSH-receptor binding assay and in transfected 
TABLE 1 | Pharmacokinetics of a single dose of subcutaneous follitropin alfa $150 \mathrm{IU}$, follitropin beta 150 IU, follitropin delta (individualized dose), follitropin epsilon 150 IU and corifollitropin alfa in healthy women (46, 50-53).

\begin{tabular}{|c|c|c|c|c|c|}
\hline Mean value & Follitropin alfa & Follitropin beta & Follitropin delta & Follitropin epsilon & Corifollitropin alfa \\
\hline $\mathrm{C}_{\max }$ & $3 \mathrm{IU} / \mathrm{L}$ & $8 \mathrm{IU} / \mathrm{L}$ & $-^{a}$ & $5.2 \mathrm{IU} / \mathrm{L}$ & $4.2 \mathrm{ng} / \mathrm{mL}$ \\
\hline$t_{\max }(\mathrm{h})$ & 16 & 12 & 10 & 22 & 44 \\
\hline Bioavailability (\%) & 74 & 77 & 64 & - & 58 \\
\hline $\mathrm{t}_{1 / 2 \beta}(\mathrm{h})$ & 37 & $40^{\mathrm{b}}(\mathrm{IM})$ & 40 & 29 & 70 \\
\hline CL (L/h) & $0.6^{c}$ & $0.01^{d}$ & 0.6 & - & 0.13 \\
\hline
\end{tabular}

${ }^{a}$ Value not reported but specified as being 1.4-fold higher than that of follitropin alfa (GONAL-f).

${ }^{b}$ Measured after intramuscular administration.

${ }^{c}$ Measured after intravenous administration.

dUnits are $\mathrm{I} / \mathrm{h} / \mathrm{kg}$.

$C L$, clearance; $C_{\max }$, maximum plasma concentration; $h$, hours; $t_{1 / 2 \beta}$, terminal elimination half-life; $t_{\max }$, time to $C_{\max }$.

Human Embryonic Kidney (HEK) 293 cells and cultured human granulosa cells (73). The differing pharmacokinetic (PK) and pharmacodynamic (PD) profiles of follitropin alfa and follitropin delta are likely to contribute to the observed differences in the properties of the two products in women, as well as to influence their efficacy for the treatment of infertility (71). In contrast with follitropin alfa, the bioactivity of follitropin delta determined by the Steelman-Pohley bioassay, which uses an international reference standard of $\mathrm{CHO}$-produced $\mathrm{r}-\mathrm{hFSH}$, does not directly predict the PD activity (71). This has been attributed to more rapid clearance of follitropin delta compared with follitropin alfa in rats, resulting in lower apparent potency (73). This means that follitropin delta cannot be dosed according to bioactivity or specific bioactivity, as other follitropins are, and is instead dosed by mass $(\mu \mathrm{g})$. Additionally, the pharmacological differences between follitropin delta and follitropin alfa suggest that these agents cannot be directly substituted in clinical practice.

The risk/benefit balance of follitropin delta was considered positive by the regulatory agencies and the most frequent adverse reactions reported during clinical trials were headache, pelvic discomfort, OHSS, pelvic pain, nausea, adnexa uteri pain and fatigue (51). In healthy female volunteers, follitropin delta demonstrated higher exposure and lower serum clearance compared with follitropin alfa (72). A phase 3 study (ESTHER-1) compared individualized doses of follitropin delta (fixed-dose throughout treatment; start dose individualized based on BMI and body weight) with follitropin alfa (starting dose of 150 IU, with potential for subsequent adjustment, with a maximum allowed daily dose of $450 \mathrm{IU}$ ) for ovarian stimulation in 1,326 women. The starting dose of follitropin delta was $12 \mu \mathrm{g}$ in patients with $\mathrm{AMH}<15 \mathrm{pmol} / \mathrm{L}$ and $0.10-0.19 \mu \mathrm{g} / \mathrm{kg}$ (maximum daily dose: $12 \mu \mathrm{g}$ ) in patients with $\mathrm{AMH} \geq 15 \mathrm{pmol} / \mathrm{L}$. This study demonstrated non-inferiority of follitropin delta to follitropin alfa for the co-primary endpoints of ongoing pregnancy rate (30.7 and 31.6\%, respectively; difference $-0.9 \%$ [95\% confidence interval (CI) $-5.9,4.1 \%])$ and ongoing implantation rate $(35.2 \%$ and $35.8 \%$, respectively; difference $-0.6 \%$ [ $95 \%$ CI $-6.1,4.8 \%]$ ), with fewer women treated with follitropin delta requiring OHSS preventative measures (74). The live birth rate was also similar with follitropin alfa and follitropin delta (29.8 and 30.7\%, respectively; difference $-0.9 \%$ [95\% CI $-5.8,4.0 \%])$. However, the initial follitropin alfa dose allowed in this study (150 IU) was at the lower end of the recommended range in the SmPC for women undergoing multifollicular development prior to ART (150-225 IU daily) (42) and this starting dose could not be individualized, whereas the dose in the follitropin delta arm was individualized according to clinical markers which reduces the comparability of outcomes (75). The EMA assessment report states that, in the ESTHER-1 trial, the non-inferiority of follitropin delta compared with follitropin alfa for ongoing pregnancy can be explained by the heterogeneity of responses in different age groups; non-inferiority was driven by the $15 \%$ of the study population aged $\geq 38$ years (75), with non-inferiority not demonstrated for women aged $\leq 37$. It has also been noted that there were a greater number of canceled cycles for poor response in the follitropin delta arm (76).

\section{Follitropin Epsilon}

Follitropin epsilon (FSH-GEX; Glycotope, Germany) is a recombinant FSH produced using a human blood cell line derived from a myeloid leukemia cell line and is currently not marketed (77). The cell lines used result in a high degree of bisecting $\mathrm{N}$-acetlyglucosamine, a high antennarity and a high degree of sialylation, in particular after enrichment of the acidic isoforms (78). In addition, follitropin epsilon is highly fucosylated and has a ratio of 2,3 to 2,6 sialylation of about $1: 1$ (78). This is different from follitropin alfa and follitropin beta, which do not have any bisecting $\mathrm{N}$-acetylgalactosamines or 2,6 sialylation. In phase 1 studies, follitropin epsilon and follitropin alfa had similar PK (Table 1), whereas PD activity (follicle growth and serum inhibin B levels) was increased with follitropin epsilon compared with follitropin alfa (77). No Phase III studies have been registered in publicly-available clinical trial repositories for this product.

\section{Corifollitropin Alfa}

Due to its short half-life FSH has to be injected daily, which may be inconvenient and an unacceptable burden to patients; longeracting $\mathrm{r}$-hFSH preparations are, therefore, being investigated (79). The only approved longer acting r-hFSH (FSH-CTP, corifollitropin alfa, Elonva; Merck Sharp Dohme, Kenilworth, NJ, USA) was developed via addition of the carbonyl-terminal 
peptide (CTP) of the $\beta$-subunit of hCG to the $\beta$-subunit of FSH, generating a chimeric protein. This prolonged the half-life of the r-hFSH without impacting on assembly with the $\alpha$-subunit, or the secretion or action of the dimer $(50,80)$. Corifollitropin alfa received marketing approval in the $\mathrm{EU}$ in 2010 for use in women undergoing fertility treatment, it is currently not approved for use in men or in the USA (41). The risk/benefit balance of corifollitropin alfa is considered positive, with the most frequently reported adverse reactions during clinical trials being pelvic discomfort, OHSS, headache, pelvic pain, nausea, fatigue and breast tenderness. Corifollitropin alfa can be administered as a single subcutaneous injection to replace the first 7 days of daily FSH therapy, simplifying treatment, as it has a 2 -fold longer half-life and almost four-fold longer time to peak serum level than other available FSH preparations (Table 1) $(81,82)$. Meta-analyses of RCTs comparing corifollitropin alfa and daily injections of r-hFSH in women receiving ART treatment found no significant differences in LBR, ongoing pregnancy rate (OPR) or clinical pregnancy rate $(\mathrm{CPR})$ between the treatments $(79,83$, 84). There was evidence of reduced LBR (co-primary endpoint) in women receiving a low dose (60 to $120 \mu \mathrm{g}$ ) of long-acting FSH compared with daily FSH (79). There was no significant increase in OHSS, however, a higher number of oocytes were stimulated with corifollitropin alfa than with r-hFSH, and there was higher cycle cancellation due to overstimulation with corifollitropin alfa $(79,83,84)$. Further research is needed to determine whether long-acting FSH is safe and effective for use in hyper-responders and poor ovarian responders and in women with all causes of subfertility (79).

Other methods for prolonging the half-life of FSH have been attempted. These include increasing elimination time by adding the Fc domain of IgG to the FSH molecule $(85,86)$, addition of new glycosylation sites and N-terminal extensions, which result in larger molecules with increased charge (87) and tethering two copies of the $\mathrm{N}$-linked glycosylation signal sequence between the $\alpha$ - and $\beta$-subunits of hFSH, creating a single-chain fusion hormone analog (88).

\section{Differences Between Recombinant and Urinary Follicle-Stimulating Hormone Preparations}

Two systematic reviews have compared r-hFSH (any preparation) with urinary gonadotropins $(89,90)$. The first compared r-hFSH with urinary gonadotropins (hMG, purified urinary FSH [u-FSH] or highly-purified u-FSH) in women undergoing ART, and included 42 trials (9,606 patients); there was no significant difference in LBR (28 trials [7,339 patients]; odds ratio $[\mathrm{OR}] 0.97,95 \% \mathrm{CI} 0.87,1.08$ ) or OHSS incidence (32 trials $[7,740$ patients]; OR $1.18,95 \%$ CI $0.86,1.61$ ) between the two types of FSH preparation (89). When only fresh cycles were considered the difference in LBR (25 trials [4,952 patients]; odds ratio $[\mathrm{OR}] 0.97,95 \%$ CI $0.85,1.11$ ) remained $(89)$. Similarly, in a comparison of r-hFSH with urinary gonadotropins (hMG or $\mathrm{u}$-FSH) for ovulation induction in patients with polycystic ovary syndrome (PCOS), there was no difference in LBR (co-primary endpoint; five trials [505 patients]; OR $1.21,95 \%$ CI $0.83,1.78$ ) or
CPR (secondary endpoint; eight trials [1,330 patients]; OR 1.05, $95 \%$ CI $0.88,1.27)$ with the two FSH preparations (90). There was also no difference in the incidence of OHSS (co-primary endpoint) between $\mathrm{r}-\mathrm{hFSH}$ and u-FSH (10 trials [1,565 patients]; OR $1.52,95 \%$ CI $0.81,2.84$ ) or between r-hFSH and hMG (two trials [52 patients]; OR 9.95, 95\% CI 0.47, 210.19) (90). Although authors of both reviews concluded that there was likely to be little if any clinical difference between r-hFSH and urinary gonadotropins $(89,90)$, authors of the latter review considered the available evidence to be of low or very low quality (90).

These results are in general agreement with those of a metaanalysis that compared r-hFSH with highly-purified hMG in ART using data from a total of 16 studies (4,040 patients) (91). When adjusted for baseline conditions, hMG treatment was associated with fewer oocytes (primary endpoint; $-2.10,95 \% \mathrm{CI}-2.83$, -1.36 ) and a higher required dose (secondary endpoint; mean difference 235 IU, 95\% CI: 16.62, 454.30) but a similar pregnancy rate (secondary endpoint; risk ratio [RR] 1.10, 95\% CI 0.97 , 1.25) (91).

These meta-analyses predominantly included fresh cycles, for example, the meta-analysis by van Wely et al. only included three trials that studied frozen-thawed embryo transfer in addition to fresh embryo transfer (89). This is because, for many years, IVF success was measured per fresh cycle or embryo transfer. As freezing and thawing technology has improved, this definition has been challenged and it has been suggested that IVF success should instead be evaluated as cumulative live birth rates (CLBR), defined as the first live birth following the use of all fresh and frozen embryos derived from a single ovarian stimulation cycle $(92,93)$. A positive correlation has been observed between live birth rate per cycle and number of oocytes retrieved, up to 15 oocytes ( $p<0.001$ for comparison between age groups) (9497). When the association between CLBR and number of oocytes was evaluated, the association remained (98-100). For example, in an analysis by Polyzos et al. the OR (95\% CI) for live birth in the second and third cycle was $1.18(1.07-1.30)$ for women with 4-9 aspirated oocytes in the first cycle, 1.41 (1.27-1.57) for women with 10-15 aspirated oocytes and 1.63 (1.42-1.88) for women with more than 15 aspirated oocytes compared with patients with $0-3$ aspirated oocytes. In several studies a greater number of oocytes were retrieved when r-hFSH rather than urinary gonadotropins were used (101). This suggests that owing to the higher number of oocytes retrieved with r-hFSH compared with urinary gonadotropins, CLBR might be higher when r-hFSH is used.

In clinical trials comparing originator follitropin alfa (GONAL-f) with highly-purified u-FSH (Metrodin HP) in women undergoing ART, the mean number of oocytes obtained with $\mathrm{r}$-hFSH was significantly higher than that obtained with u-FSH $(102,103)$. There was no difference in CPR (secondary endpoint; 45 and $48 \%$, respectively) (102) or LBR (secondary endpoint; 36 and $36 \%$, respectively) (103), but singleton pregnancies were more common with u-FSH $(102,103)$. When follitropin beta (Puregon) and highly-purified u-FSH (Metrodin HP) were compared in women undergoing IVF, the mean number of oocytes (primary endpoint; 9.7 vs. $8.9 ; 95 \%$ CI for 
the difference: $-1.7,3.2$ ) and CPRs did not differ significantly between treatment groups (secondary endpoints; per attempt, 35.4 vs. $26.6 \%$, respectively [ $95 \%$ CI for the difference: -12.1 , 29.6]; per transfer, 40.8 vs. $28.6 \%$, respectively [ $95 \%$ CI for the difference: $-10.3,34.8]$ ) (104).

\section{r-hFSH for the Treatment of Male Infertility}

FSH plays an important role in spermatogenesis, stimulating the Sertoli cells to facilitate germ cell differentiation. Follitropin alfa and follitropin beta are approved for clinical use in males who have congenital or acquired hypogonadotropic hypogonadism, for the stimulation of spermatogenesis with concomitant hCG therapy $(42,46)$. In a small study $(N=8), \mathrm{r}-\mathrm{hFSH}$ (follitropin alfa) was observed to induce testicular growth, spermatogenesis and fertility, with acceptable tolerability, in men with gonadotropin deficiency; the magnitude of effect was considered to be similar to that achieved historically with u-FSH when used to restore normal fertility in men with gonadotropin deficiency (105). In a second larger study, 15 of 19 men treated with r-hFSH and hCG achieved spermatogenesis (106). A Cochrane review evaluating gonadotropins for idiopathic male factor subfertility, identified six RCTs including 456 patients, and observed a higher spontaneous pregnancy rate per couple with gonadotropin treatment compared with placebo/no treatment (five studies [412 patients]; OR 4.94, 95\% CI 2.13, 11.44) (107). This review noted that reporting of adverse event data was sparse. However, the risk/benefit balance in males is considered positive (40).

\section{Human Chorionic Gonadotropin}

Recombinant hCG ( $\mathrm{r}-\mathrm{hCG})$ is produced in a CHO cell line in a similar manner to $\mathrm{r}$-hFSH $(55,108)$ and is suitable for subcutaneous injection and self-administration (109). In healthy subjects, the PK (Table 2) and PD profiles of r-hCG are consistent with endogenous hCG physiology and similar to those seen with urinary hCG (u-hCG) (111). The elimination half-lives of r-hCG and u-hCG are comparable (29-30 h for r-hCG $250 \mu \mathrm{g}$ vs. $35 \mathrm{~h}$ for $\mathrm{u}$-hCG $5000 \mathrm{IU}$ ) as are the areas under the concentration-time curve; however, u-hCG tends to be distributed and eliminated slightly slower than r-hCG (111).

In the late 1990s, Duffy et al. observed that r-hCG and $\mathrm{u}$-hCG were equally effective for stimulating steroidogenic and peptidergic activities of the corpus luteum during simulated early pregnancy in rhesus monkeys (112). The equipotency of r-hCG and $\mathrm{u}$-hCG was also demonstrated in macaque monkeys, with the numbers of oocytes resuming meiosis and undergoing IVF being similar in animals treated with either the recombinant or urinary product (113). However, the bioactivity of r-hCG was greater than that of $\mathrm{u}-\mathrm{hCG}$, when administered at the same dose (measured in IU), as determined by a mouse Leydig cell bioassay validated for macaque serum $(p<0.05)$ (113). Subsequently, in 2001, r-hCG (choriogonadotropin alfa, Ovitrelle; Merck KGaA, Darmstadt, Germany) was licensed for clinical use as a trigger for final follicular maturation/ovulation and luteinisation after stimulation of follicular growth (109).

Three randomized, double-blind, double-dummy, parallelgroup, multicentre trials have confirmed the similar efficacy of
TABLE 2 | Pharmacokinetics of a single dose of subcutaneous choriogonadotropin alfa ( $r$-hCG; dose and population not reported) and lutropin alfa (r-hLH) $75 \mathrm{IU}$ to 40,000 IU in female volunteers $(109,110)$.

\begin{tabular}{lcc}
\hline Mean value & r-hCG & r-hLH \\
\hline Bioavailability (\%) & 40 & 60 \\
$\mathrm{t}_{1 / 2 \beta}(\mathrm{h})$ & 30 & $\approx 10-12$ \\
$\mathrm{CL}(\mathrm{L} / \mathrm{h})$ & $0.2^{\mathrm{a}}$ & 2 \\
\hline
\end{tabular}

a Measured after intravenous administration.

$C L$, clearance, $C_{\max }$, maximum plasma concentration; $h$, hours; $t_{1 / 2 \beta}$, terminal elimination half-life; $t_{\max }$, time to $C_{\max }$.

r-hCG and u-hCG. In one, there were no observed differences following treatment with $\mathrm{r}-\mathrm{hCG}$ or $\mathrm{u}-\mathrm{hCG}$ in the number of oocytes retrieved (primary endpoint; mean \pm standard deviation [SD] $10.8 \pm 4.5$ vs. $10.3 \pm 5.1$ ) or the number of patients pregnant (secondary endpoint; 10 in each group) and adverse events were generally mild or moderate among the 84 women undergoing IVF or intracytoplasmic sperm injection (ICSI) and embryo transfer (114). Similarly, a multinational study in anovulatory or oligo-ovulatory patients showed that r-hCG administration resulted in the same rates of ovulation and pregnancy as u-hCG administration (115). Overall, 162 of the 177 patients $(91.5 \%)$ in the per protocol population ovulated (primary endpoint): 95.3\% receiving $\mathrm{r}-\mathrm{hCG}$ and $88.0 \%$ receiving $\mathrm{u}-\mathrm{hCG}$; however, in this study, r-hCG was better tolerated than u-hCG (115). The European Recombinant Human Chorionic Gonadotropin Study Group compared the efficacy and safety of r-hCG and u-hCG for inducing final follicular maturation and early luteinisation in 172 evaluable women undergoing ovulation induction for ART (116). The primary endpoint, the mean number of oocytes retrieved per patient was not significant between treatments (11.6 for r-HCG and 10.6 for u-hCG; two-sided $90 \%$ CI for the difference: $-0.841,1.515)$. Patients treated with $\mathrm{r}$-hCG demonstrated better outcomes for number of mature oocytes (9.4 and 7.1 with rhCG and u-hCG, respectively; $p=0.027$ ), serum progesterone (day 1 post hCG administration: 30.1 vs. $23.3 \mathrm{nmol} / \mathrm{L}[p=0.04]$; day 6-7 post hCG administration: 391.9 vs. $315.9 \mathrm{nmol} / \mathrm{L}[p$ $=0.03]$ ) and hCG (day of embryo transfer: $2.1 \mu \mathrm{g} / \mathrm{L}$ vs. 1.6 $\mu \mathrm{g} / \mathrm{L}[p=0.0001])$ levels, CPR (32 [33.0\%] and 23 [24.7\%] with r-hCG, and u-hCG, respectively), and LBR (26 [26.8\%] and 21 [22.6\%] with r-hCG and u-hCG, respectively). While both treatments were well tolerated, the incidence of adverse events was significantly higher in patients treated with u-hCG. Injection site reactions being the most common adverse events in with both treatments in these latter two studies $(115,116)$. Investigators concluded, that for triggering ovulation, $\mathrm{r}-\mathrm{hCG}$ may have significant advantages over u-hCG (116).

Treatment with $\mathrm{r}$-hCG and $\mathrm{u}$-hCG was also shown to result in similar numbers of oocytes (primary endpoint) and $2 \mathrm{PN}$ oocytes (secondary endpoint) obtained in a prospective, open, randomized study in 275 women requiring induction of final follicular maturation and luteinisation for IVF with embryo transfer (117). In this study, the tolerability of r-hCG and u-hCG was similar, with $>95 \%$ of injections with either hCG producing no adverse reactions. More recently, Bellavia et al. 
reported that highly purified u-hCG was not inferior to $\mathrm{r}-\mathrm{hCG}$ with regard to the mean number of oocytes retrieved (13.3 vs. $12.5)$, with no differences observed in fertilization rate $(57.3 \%$ [467/815] vs. $61.3 \%$ [482/787]) or tolerability between the hCG preparations (118).

\section{Luteinizing Hormone}

Recombinant human luteinizing hormone ( $\mathrm{r}-\mathrm{hLH}$, lutropin alfa, Luveris; Merck KGaA, Darmstadt, Germany) received marketing authorization for clinical use in 2000 in Europe and 2004 in the US (subsequently withdrawn at Merck KGaA's request in 2016) (110). r-hLH is produced in a similar manner to FSH in $\mathrm{CHO}$ cells transfected with vectors encoding the $\alpha$ and $\beta$ subunits (119), and is suitable for subcutaneous injection and self-administration (110). The PK of r-hLH is almost identical to the LH component of hMG (Pergonal; Laboratoires Serono, Aubonne, Switzerland) with a terminal half-life of $\sim 10-12 \mathrm{~h}$ (Table 2) (120). It should be highlighted that at the time of this analysis the LH component of hMG preparations was predominantly the LH component of post-menopausal urine, rather than hCG as is more common in later and currently available more highly purified preparations. r-hLH is approved for use in women with severe LH and FSH deficiency, in combination with r-hFSH (121). In specific countries outside Europe (Russia, Mexico) r-hLH is also approved for patients with suboptimal ovarian response in the context of ART treatment (122). To improve convenience, a 2:1 fixed-ratio combination of r-hFSH and r-hLH has also been developed (Pergoveris; Merck KGaA, Darmstadt, Germany), which received marketing approval in Europe in 2007 (123). Pergoveris is not currently approved in the US.

In women with severe FSH and LH deficiency, r-hLH has been shown to support r-hFSH-induced follicular development (124, 125). In an open-label, dose-finding study, in which women with hypogonadotropic hypogonadism were randomized to receive $\mathrm{r}-$ hLH in combination with r-hFSH $150 \mathrm{IU}, 0,14.3,66.7$, and 88.0\% of women treated with r-hLH 0 IU $(n=8), 25 \mathrm{IU}(n=7), 75 \mathrm{IU}$ $(n=9)$ and $225 \mathrm{IU}(n=10)$, respectively, had good or excessive follicular growth ( $p<0.01$ by Cochran-Armitage trend test for difference between groups) (125). This study demonstrated that although $\mathrm{LH}$ requirements varied, a minimum effective daily dose of 75 IU provides adequate follicular development and steroidogenesis. A second study confirmed that r-hFSH $150 \mathrm{IU}$ plus $\mathrm{r}$-hLH $75 \mathrm{IU}$ is the most appropriate dose schedule for hypogonadotropic anovulatory women, with sufficient follicular growth observed in $94 \%(79 / 84)$ of initiated cycles (five cycles in three patients required a dose increase) and pregnancy achieved by 15 of the 38 treated women (39.5\%) (126). A study in 169 women aged 38-42 years randomized to receive a combination of r-hFSH:r-hLH in one of four ratios: 1:0, 1:1, 2:1, or $3: 1$ (127). The starting dose of r-hFSH was $225 \mathrm{IU}$, with r-hLH dosed according to the ratio, and the dose of r-hFSH could be adjusted up to $450 \mathrm{IU}$. A greater mean number of oocytes was retrieved in the group receiving 2:1 r-hFSH:r-hLH compared with those receiving 1:1 and 3:1 r-hFSH:r-hLH (8.4, 7.4, and 7.5 , respectively), and the adjusted clinical pregnancy rate was higher in the groups receiving 3:1 or 2:1 r-hFSH:r-hLH (12.2 and $12.0 \%$, respectively) compared with those receiving 1:0 and 1:1 r-hFSH:r-hLH (4.6 and 2.4\%, respectively).The 2:1 fixed-ratio is supported by the dose-finding and confirmatory studies in women with hypogonadotropic hypogonadism, as well as the ART study summarized here.

The ESPART study was an RCT evaluating the effect of fixedratio (2:1) combination r-hFSH:r-hLH compared with r-hFSH alone for controlled ovarian stimulation in 939 women with POR $(128,129)$. In the ESPART study, to be defined as having POR, women had to meet at least two of the following criteria: advanced maternal age ( $\geq 40-<41$ years); a previous ART cycle with $\leq 3$ oocytes retrieved with a conventional stimulation protocol; an abnormal ovarian reserve test characterized by an AMH level between 0.12 and $1.3 \mathrm{ng} / \mathrm{ml}$, inclusive. There were no differences observed in efficacy outcomes (number of oocytes retrieved [primary endpoint]; biochemical pregnancy rate, $\mathrm{CPR}$, OPR; and LBR) between patients receiving $\mathrm{r}-\mathrm{hFSH} / \mathrm{r}-\mathrm{hLH}$ and those receiving $\mathrm{r}$-hFSH alone. However, a post-hoc analysis of the ESPART study observed a higher live birth rate with $r-$ hLH supplementation in patients with moderate or severe POR, while a higher live birth rate was observed with $\mathrm{r}$-hFSH alone in patients with mild POR (130).

Five recent meta-analyses have evaluated whether supplementation of FSH with LH for controlled ovarian stimulation (COS) might improve ART outcomes (131-135). While LBR is the preferred outcome, reality has shown that LBR is only reported in a small proportion of available studies, and most papers report intermediate pregnancy outcomes (such as CPR or OPR), representing relevant outcomes to measure clinical treatments benefits in reproductive medicine when pregnancy losses are not impacted (136-139). These meta-analyses have reported some conflicting results, despite there being overlap among the studies included.

These meta-analyses have relied on RCTs conducted in the general population, and either suggest that there is no beneficial effect from LH supplementation or that $\mathrm{LH}$ supplementation to $\mathrm{FSH}$ results in improvements in some outcomes in these patients. A higher number of oocytes were retrieved without LH supplementation (primary endpoint; 29 studies [5,840 patients] standard mean difference $-0.20,95 \%$ CI $-0.38,-0.02 ; p=$ 0.03 ) in one meta-analysis (135), whereas no difference in this endpoint was observed in another meta-analysis (primary endpoint; 43 studies [6,341 patients]; RR 1.17, 95\% CI 0.42, 1.92; $p$ $=0.002$ ) (133). A higher pregnancy rate (secondary endpoint; 29 studies [5,565 patients] OR 1.20, 95\% CI 1.06, 1.37) was observed by one meta-analysis (135), whereas in other meta-analyses a higher CPR (secondary endpoint; 43 studies [6,393 patients]; RR $1.3,95 \%$ CI 1.05, 1.62; $p=0.016$ ) (133), higher OPR (secondary endpoint: 19 studies [3,129 patients] OR 1.20, 95\% CI 1.01, 1.42) (134) and higher LBR (primary endpoint: 4 studies [499 patients] OR 1.32, 95\% CI 0.85, 2.06; secondary endpoint: 39 studies [6,237 patients] RR 1.11, 95\% CI 1.01, 1.21) $(133,134)$ were observed with LH supplementation to FSH compared with FSH alone. These findings may reflect the different characteristics of the pooled populations, depending on the trials included.

It has been suggested that the benefits of LH supplementation may occur in subpopulations characterized by LH insufficiency, 
including hypo-responders $(133,134)$. Hypo-response is characterized by an unexpected resistance to ovarian stimulation with standard doses of gonadotropins. This resistance might be diagnosed in women with otherwise normal ovarian reserve during ovarian stimulation who demonstrate an initial slow response and observed through serum estradiol levels and follicular growth or diagnosed retrospectively where higherthan-expected gonadotropin doses have been used (140). In patients with poor ovarian response (POR; including hyporesponders), supplementation with $\mathrm{LH}$ results in increased CPR (post-hoc analysis: RR 1.3, 95\% CI 1.05, 1.62; p=0.016) (133), OPR (subgroup analysis: 3 trials [79 patients] OR 2.06, 95\% CI 1.20, 3.53) (134) and LBR (post-hoc analysis: RR 1.30, 95\% CI $0.95,1.78)(133)$.

When only hypo-responders were considered, supplementation with $\mathrm{LH}$ did not increase the number of oocytes retrieved (two RCTs and one cohort study [319 patients] OR 1.98, 95\% CI $0.17,3.80 ; p=0.03$ ), but did increase implantation rate (four RCTS and one cohort study [766 patients] OR 2.62, 95\% CI 1.37, 4.99; $p=0.004$ ), and CPR (three RCTs and one cohort study [361 patients] OR 2.03, 95\% CI 1.27, $3.25 ; p=0.003$ ) (132) compared with FSH alone. LBR could not be evaluated by this meta-analysis as it was only included as an endpoint in one study (132).

A systematic review (without meta-analysis) that assessed the effect of $\mathrm{r}-\mathrm{hLH}$ supplementation in COS as part of ART in six different patient populations (prevention of OHSS; women with profoundly suppressed LH levels after administration of a gonadotropin-releasing hormone [GnRH] agonist; women cotreated with a GnRH antagonist; women with a hypo-response to r-hFSH; women of advanced reproductive age; and women with POR, including women meeting the ESHRE Bologna criteria) identified two populations that may benefit from this treatment approach (131). In women with a hypo-response to r-hFSH the evaluated literature suggests that a greater number of oocytes might be retrieved and a higher implantation rate obtained with LH plus FSH compared with FSH alone (based on two studies). In women of advance reproductive age a higher implantation rate may be obtained with LH plus FSH compared with FSH alone (based on four studies). A lower proportion of patients with OHSS were observed with LH supplementation in patients when used for prevention of OHSS. No difference between treatment with $\mathrm{LH}$ plus FSH and FSH alone was observed in women with profoundly suppressed LH levels after administration of a $\mathrm{GnRH}$ agonist, women co-treated with a $\mathrm{GnRH}$ antagonist and poor ovarian responders.

\section{ORAL GONADOTROPINS}

All gonadotropin preparations have to be injected, which increases the treatment burden for patients. There has therefore been interest in producing a product that can be dosed orally. It is not possible to dose gonadotropins orally because they are proteins and will not be absorbed, rather they are digested by enzymes. As a result of this, attempts to produce an oral drug for ovarian stimulation have focussed on FSH agonists. One oral
FSH agonist has been evaluated in healthy females but no effect on follicular development was observed, which was eventually attributed to the low doses used (141). Non-conclusive data is available for this option nowadays.

\section{INJECTION DEVICES}

Animal-derived and urinary gonadotropin products had to be injected intramuscularly using a syringe and vial, with reconstitution required before injection. Owing to the increased purity of recombinant products, a smaller injection volume is required and these can be injected subcutaneously using smaller gauge needles. In addition, these products have greater stability and liquid formulations of recombinant products have been produced, removing the need for reconstitution before injection. This in turn has enabled the development of pen injection devices, which are designed to improve ease-of-use and patient convenience, including the ability to both select the starting dose with greater precision (in increments as low as 12.5 IU) and adapt the dose during treatment, based on treatment response in small increments (12.5 IU) (142-144).

\section{CONCLUSIONS}

ART has come a long way since 1927, when gonadotropins were first identified, and currently available gonadotropin preparations better enable treatment individualization as part of patient-centered care. Patient-centeredness should be an aspect of all consultations and treatment decisions relating to medically assisted reproduction treatment. This should include discussions of whether treatment is appropriate, and if it is appropriate, which treatment would be most favorable. This treatment should be individualized according to the characteristics of the patient(s) and monitored to ensure that effectiveness is optimal, based on treatment response and safety, with treatment adjusted during treatment if it is not. The availability of recombinant products, which provide a pure form of the gonadotropin and can be accurately dosed, has improved the ability of medical practitioners to individualize treatment in this manner. Currently available products can be injected subcutaneously rather than intramuscularly, and pen injection devices are available, improving ease-of-use and more precise dose selection and adaption (in 12.5 IU dose increments). Work to develop new preparations is continuing, and a goal must remain the development of orally active FSH agonists and antagonists.

\section{AUTHOR CONTRIBUTIONS}

All authors listed have made a substantial, direct and intellectual contribution to the work, and approved it for publication.

\section{ACKNOWLEDGMENTS}

Medical writing support was provided by Alexander Jones of inScience Communications, Springer Healthcare, and was funded by Merck KGaA, Darmstadt, Germany. 


\section{REFERENCES}

1. Ascheim S, Zondek B. Hypophysenvorderlappenhormone und ovarialhormone im harn von schwangeren. Klin Wochenschr. (1927) 6:13. doi: $10.1007 / \mathrm{BF} 01728562$

2. Lunenfeld B, Ezcurra D, D'Hooghe T. The development and evolution of gonadotropins in ART. Fertil Steril. (2018) 110:255-62. doi: 10.1016/j.fertnstert.2018.06.005

3. Seegar-Jones GE, Gey GO, Ghisletta M. Hormone production by placental cells maintained in continuous culture. Bull Johns Hopkins Hosp. (1943) 72:26-38.

4. Lunenfeld B. Gonadotropin stimulation: past, present and future. Reprod Med Biol. (2012) 11:11-25. doi: 10.1007/s12522-011-0097-2

5. Smith PE, Engle ET. Experimental evidence of the role of anterior pituitary in development and regulation of gonads. Am J Anat. (1927) 40:159. doi: 10.1002/aja.1000400202

6. Zondek B. Weitere untersuchungen zur darstellung, biologie und klinik des hypophysenvorderlappenhormones (Prolan). Zentralblatt fur Gynakologie. (1929) 14:834-48.

7. Lunenfeld B. Historical perspectives in gonadotrophin therapy. Hum Reprod Update. (2004) 10:453-67. doi: 10.1093/humupd/dmh044

8. D'Amour FE, D'Amour MC. The biologic potency of international standrad chorionic gonadotropin. Endocrinology. (1940) 26:93-6. doi: 10.1210/endo-26-1-93

9. Gurin S, Bachman G, Wilson DW. The gonadotropic hormone of urine of pregnancy. ii) Chemical studies of preparations having high biological activity. J Biol Chem. (1940) 133:467.

10. Katzman PA, Godfried M, Cain CK, Doisy EA. The preparation of chorionic gonadotrophin by chromatographic adsorption. J Chem Biol. (1943) 148:501-7.

11. Greep RO, Van Dyke HB, Chan BF. Gonadotropins of the swine pituitary: various biological effects of purified thylakentrin (FSH) and pure metakentrin (ICSH). Endocrinolology. (1942) 30:635-49. doi: 10.1210/endo-30-5-635

12. Steelman SL, Lamont WA, Baltes BJ. Preparation of highly active follicle stimulating hormone from swine pituitaries. Endocrinology. (1955) 56:216-7. doi: 10.1210/endo-56-2-216

13. Cole HH, Hart GH. The potency of blood serum of mares in progressive stages of pregnancy in affecting the sexual maturity of the immature rat. Am J Physiol. (1930) 93:57. doi: 10.1152/ajplegacy.1930.93.1.57

14. Mazer C, Ravetz E. The effect of combined administration of chorionic gonadotropin and the pituitary synergist on the human ovary. Am J Obstet Gynaecol. (1941) 41:474-588. doi: 10.1016/S0002-9378(41)90825-6

15. Leathem JH, Rakoff A. Gonadotrophic hormone therapy in man complicated by antihormone formation. Am J Obstet Gynecol. (1948) 56:521-6. doi: 10.1016/0002-9378(48)9 0638-3

16. Gemzell C. Human pituitary gonadotropins in the treatment of sterility. Fertil Steril. (1966) 17:149-59. doi: 10.1016/S0015-0282(16)35880-0

17. Gemzell CA, Diczfalusy E, Tillinger G. Clinical effect of human pituitary follicle-stimulating hormone (FSH). J Clin Endocrinol Metab. (1958) 18:1333-48. doi: 10.1210/jcem-18-12-1333

18. Cochius JI, Burns RJ, Blumbergs PC, Mack K, Alderman CP. CreutzfeldtJakob disease in a recipient of human pituitary-derived gonadotrophin. Aust NZ J Med. (1990) 20:592-3. doi: 10.1111/j.1445-5994.1990.tb01322.x

19. Cochius JI, Hyman N, Esiri MM. Creutzfeldt-Jakob disease in a recipient of human pituitary-derived gonadotrophin: a second case. J Neurol Neurosurg Psychiatry. (1992) 55:1094-5. doi: 10.1136/jnnp.55.11.1094

20. Dumble LJ, Klein RD. Creutzfeldt-Jakob legacy for Australian women treated with human pituitary gonadotropins. Lancet. (1992) 340:847-8. doi: 10.1016/0140-6736(92)92720-Z

21. Borth $\mathrm{R}$, Lunenfeld $\mathrm{B}$, de Watteville $\mathrm{H}$. Active gonadotrope d'un extrait d'urines de femmes en menopause. Experientia. (1954) 10:266-8. doi: 10.1007/BF02157401

22. Albert A. Human Pituitary Gonadotropins- a workshop conference. In: Charles C Thomas, December 1959. Gatlinburg, TN: Springfield (1961).

23. Borth R, Lunenfeld B, De Watteville H. Day-to-day variation in urinary gonadotrophin and steroid levels during the normal menstrual cycle. Fertil Steril. (1957) 8:233-54. doi: 10.1016/S0015-0282(16) 61356-0

24. Lunenfeld B, Menzi A, Volet B. Clinical effects of human postmenopausal gonadotropin. In: Fuchs F, editor. Advance Abstracts of Short Communications, 1st International Congress of Endocrinology. Copenhagen: Periodical (1960).

25. Lunenfeld B, Rabau E, Rumney G, Winkelsberg G. The responsiveness of the human ovary to gonadotropin (Hypophysis III). Proc Third World Cong Gynecol Obstet. (1961) 1:22.

26. Lunenfeld B, Sukmovici S, Rabau E, Eshkol A. L'induction de l'ovulation dans les amenorrees hypophysaires par un traitement combini de gonadotrophines unnaires minopausiques et de gonadotrophines chononiques. CR Soc FT Gynecol. (1962) 5:1-6.

27. Lunenfeld B. Treatment of anovulation by human gonadotropins. $J$ Int Fed Gynecol Obstet. (1963) 1:15. doi: 10.1002/j.1879-3479.1963.tb 00335.x

28. World Health Organization. Expert Committee on Biological Standardization (Chair B.Lunenfeld), Vol 565. Technical Report Series. Geneva: World Health Organization (1972).

29. Steelman SL, Pohley FM. Assay of the follicle stimulating hormone based on the augmentation with human chorionic gonadotropin. Endocrinology. (1953) 53:604-16. doi: 10.1210/endo-53-6-604

30. WHO Expert Committee. Agents Stimulating Gonadal Function in Human (Chair B. Lunenfeld). Technical Report Series. WHO (1973).

31. Steptoe PC, Edwards RG. Birth after the reimplantation of a human embryo. Lancet. (1978) 2:366. doi: 10.1016/S0140-6736(78)92957-4

32. Inge GB, Brinsden PR, Elder KT. Oocyte number per live birth in IVF: were steptoe and edwards less wasteful? Hum Reprod. (2005) 20:588-92. doi: 10.1093/humrep/deh655

33. Jones HW Jr. The use of controlled ovarian hyperstimulation $(\mathrm{COH})$ in clinical in vitro fertilization: the role of georgeanna seegar jones. Fertil Steril. (2008) 90:e1-3. doi: 10.1016/j.fertnstert.2007. 07.1333

34. Jones HW Jr, Jones GS, Andrews MC, Acosta A, Bundren C, Garcia J, et al. The program for in vitro fertilization at Norfolk. Fertil steril. (1982) 38:14-21. doi: 10.1016/S0015-0282(16)46390-9

35. Donini P, Puzzuoli D, D’Alessio I, Lunenfeld B, Eshkol A, Parlow AF. Purification and separation of follicle stimulating hormone (FSH) and luteinizing hormone (LH) from human postmenopausal gonadotrophin (HMG). II. Preparation of biological apparently pure FSH by selective binding of the LH with an anti-HGG serum and subsequent chromatography. Acta Endocrinol. (1966) 52:186-98. doi: 10.1530/acta.0.0520186

36. Ferring Pharmaceuticals Ltd. Menopur 75IU. (2015). Available online at: https://www.medicines.org.uk/emc/medicine/4322 (accessed March 28, 2019).

37. Howles C, Tanaka T, Matsuda T. Management of male hypogonadotrophic hypogonadism. Endocr J. (2007) 54:177-90. doi: 10.1507/endocrj.02-KR-98

38. Lunenfeld B, Mor A, Mani M. Treatment of male infertility. I. human gonadotropins. Fertil Steril. (1967) 18:581-92. doi: 10.1016/S0015-0282(16)36421-4

39. Macleod J, Pazianos A, Ray BS. Restoration of human spermatogenesis by menopausal gonadotrophins. Lancet. (1964) 1:1196-7. doi: 10.1016/S0140-6736(64)91212-7

40. European Medicines Agency. GONAL-f. (2018). Available online at: https:// www.ema.europa.eu/en/medicines/human/EPAR/gonal-f (accessed January 29, 2019).

41. Merck Sharp Dohme Limited. Elonva. (2019) Available online at: https:// www.ema.europa.eu/en/medicines/human/EPAR/elonva (accessed March 26, 2019).

42. EMD Serono I. Gonal-F. (2018). Available online at: https://www.drugs.com/ pro/gonal-f.html (accessed 201926 Mar 2019).

43. European Medicines Agency. Ovaleap. (2018). Available online at: https:// www.ema.europa.eu/en/medicines/human/EPAR/ovaleap (accessed January 29, 2019).

44. European Medicines Agency. Bemfola. (2018). Available online at: https://www.ema.europa.eu/en/medicines/human/EPAR/bemfola (accessed January 29, 2019). 
45. Drugs.com. Follistim AQ Approval History. (2018). Available online at: https://www.drugs.com/history/follistim-aq.html (accessed March 26, 2019).

46. European Medicines Agency. Puregon. (2018). Available online at: https://www.ema.europa.eu/en/medicines/human/EPAR/puregon (accessed January 29, 2019).

47. Howles CM. Genetic engineering of human FSH (Gonal-F). Hum Reprod Update. (1996) 2:172-91. doi: 10.1093/humupd/2.2.172

48. Olijve W, de Boer W, Mulders JW, van Wezenbeek PM. Molecular biology and biochemistry of human recombinant follicle stimulating hormone (Puregon). Mol Hum Reprod. (1996) 2:371-82. doi: 10.1093/molehr/ 2.5.371

49. Goa KL, Wagstaff AJ. Follitropin alpha in infertility: a review. BioDrugs. (1998) 9:235-60. doi: 10.2165/00063030-199809030-00006

50. European Medicines Agency. Elonva: Corifollitropin Alfa. (2015). Available online at: https://www.ema.europa.eu/en/medicines/human/EPAR/elonva (accessed March 10, 2019).

51. European Medicines Agency. Rekovelle: Follitropin Delta. (2017). Available online at: http://www.ema.europa.eu/ema/index.jsp?curl= pages/medicines/human/medicines/003994/human_med_002044.jsp\& mid=WC0b01ac058001d124 (accessed June 21, 2018).

52. le Contonnec JY, Porchet HC, Beltrami V, Khan A, Toon S, Rowland M. Clinical pharmacology of recombinant human follicle-stimulating hormone. II. single doses and steady state pharmacokinetics. Fertil Steril. (1994) 61:679-86. doi: 10.1016/S0015-0282(16)56645-X

53. Voortman G, Mannaerts BM, Huisman JA. A dose proportionality study of subcutaneously and intramuscularly administered recombinant human follicle-stimulating hormone (Follistim*/Puregon) in healthy female volunteers. Fertil Steril. (2000) 73:1187-93. doi: 10.1016/S0015-0282(00)00542-2

54. de Leeuw R, Mulders J, Voortman G, Rombout F, Damm J, Kloosterboer L. Structure-function relationship of recombinant follicle stimulating hormone (Puregon). Mol Hum Reproduct. (1996) 2:361-9. doi: $10.1093 / \mathrm{molehr} / 2.5 .361$

55. Leao Rde B, Esteves SC. Gonadotropin therapy in assisted reproduction: an evolutionary perspective from biologics to biotech. Clinics. (2014) 69:279-93. doi: 10.6061/clinics/2014(04)10

56. Bassett RM, Driebergen R. Continued improvements in the quality and consistency of follitropin alfa, recombinant human FSH. Reprod Biomed Online. (2005) 10:169-77. doi: 10.1016/S1472-6483(10)60937-6

57. Hugues J-N, Durnerin IC. Gonadotrophins - filled-by-mass versus filled-by-bioassay. Reprod Biomed Online. (2005) 10:11-7. doi: 10.1016/S1472-6483(11)60385-4

58. Brinsden P, Akagbosu F, Gibbons LM, Lancaster S, Gourdon D, Engrand $\mathrm{P}$, et al. A comparison of the efficacy and tolerability of two recombinant human follicle-stimulating hormone preparations in patients undergoing in vitro fertilization-embryo transfer. Fertil Steril. (2000) 73:114-6. doi: 10.1016/S0015-0282(99)00450-1

59. Harlin J, Csemiczky G, Wramsby H, Fried G. Recombinant follicle stimulating hormone in in-vitro fertilization treatment-clinical experience with follitropin alpha and follitropin beta. Hum Reprod. (2000) 15:239-44. doi: 10.1093/humrep/15.2.239

60. Tulppala M, Aho M, Tuuri T, Vilska S, Foudila T, Hakala-Ala-Pietila T, et al. Comparison of two recombinant follicle-stimulating hormone preparations in in-vitro fertilization: a randomized clinical study. Hum Reprod. (1999) 14:2709-15. doi: 10.1093/humrep/14.11.2709

61. Williams RS, Vensel T, Sistrom CL, Kipersztok S, Rhoton-Vlasak A, Drury K. Pregnancy rates in varying age groups after in vitro fertilization: a comparison of follitropin alfa (Gonal F) and follitropin beta (Follistim). Am J Obstet Gynecol. (2003) 189:342-6. doi: 10.1067/S0002-9378(03)00728-2

62. Mastrangeli R, Satwekar A, Cutillo F, Ciampolillo C, Palinsky W, Longobardi S. In-vivo biological activity and glycosylation analysis of a biosimilar recombinant human follicle-stimulating hormone product (Bemfola) compared with its reference medicinal product (GONAL-f). PLoS ONE. (2017) 12:e0184139. doi: 10.1371/journal.pone.0184139

63. de Mora F, Fauser BCJM. Biosimilars to recombinant human FSH medicines: comparable efficacy and safety to the original biologic. Reprod Biomed Online. (2017) 35:81-6. doi: 10.1016/j.rbmo.2017.03.020
64. Winstel R, Wieland J, Gertz B, Mueller A, Allgaier H. Manufacturing of recombinant human follicle-stimulating hormone ovaleap ((R)) (XM17), comparability with gonal-f((R)), and performance/consistency. Drugs $R D$. (2017) 17:305-12. doi: 10.1007/s40268-017-0182-Z

65. Orvieto R, Seifer DB. Biosimilar FSH preparations- are they identical twins or just siblings? Reprod Biol Endocrinol. (2016) 14:32. doi: 10.1186/s12958-016-0167-8

66. European Medicines Agency. Biosimilars in the EU. (2017). Available online at: https://www.ema.europa.eu/documents/leaflet/biosimilars-euinformation-guide-healthcare-professionals_en.pdf (accessed January 30, 2019).

67. Rettenbacher M, Andersen AN, Garcia-Velasco JA, Sator M, Barri P, Lindenberg $\mathrm{S}$, et al. A multi-centre phase 3 study comparing efficacy and safety of Bemfola $((\mathrm{R}))$ versus Gonal- $\mathrm{f}(\mathrm{R})$ ) in women undergoing ovarian stimulation for IVF. Reprod Biomed Online. (2015) 30:504-13. doi: 10.1016/j.rbmo.2015.01.005

68. Strowitzki T, Kuczynski W, Mueller A, Bias P. Randomized, activecontrolled, comparative phase 3 efficacy and safety equivalence trial of Ovaleap(R) (recombinant human follicle-stimulating hormone) in infertile women using assisted reproduction technology (ART). Reprod Biol Endocrinol. (2016) 14:1. doi: 10.1186/s12958-015-0135-8

69. Papsch R, Roeder C, D’Hooghe T, Longobardi S. PMU40 - live birth rate (LBR), ongoing pregnancy rate (OPR) and ovarian hyperstimulation syndrome (OHSS) risk with originator versus biosimilar recombinant follitropin ALFA: a pooled analysis of clinical trial data. Value Health. (2018) 21:S314-S5. doi: 10.1016/j.jval.2018.09.1876

70. Strowitzki T, Kuczynski W, Mueller A, Bias P. Safety and efficacy of Ovaleap(R) (recombinant human follicle-stimulating hormone) for up to 3 cycles in infertile women using assisted reproductive technology: a phase 3 open-label follow-up to Main Study. Reprod Biol Endocrinol. (2016) 14:31. doi: 10.1186/s12958-016-0164-y

71. Koechling W, Plaksin D, Croston GE, Jeppesen JV, Macklon KT, Andersen CY. Comparative pharmacology of a new recombinant FSH expressed by a human cell line. Endocrine Connect. (2017) 6:297-305. doi: 10.1530/EC-17-0067

72. Olsson H, Sandstrom R, Grundemar L. Different pharmacokinetic and pharmacodynamic properties of recombinant follicle-stimulating hormone (rFSH) derived from a human cell line compared with rFSH from a non-human cell line. J Clin Pharmacol. (2014) 54:1299-307. doi: 10.1002/ jcph.328

73. Therapeutic Goods Administration (TGA) Commonwealth of Australia. Australian Public Assessment Report (AusPAR) Rekovelle. (2017). Available online at: https://www.tga.gov.au/sites/default/files/auspar-follitropindelta-rhu-171025.docx (accessed March 29, 2019).

74. Nyboe Andersen A, Nelson SM, Fauser BCJM, García-Velasco JA, Klein $\mathrm{BM}$, Arce J-C, et al. Individualized versus conventional ovarian stimulation for in vitro fertilization: a multicenter, randomized, controlled, assessorblinded, phase 3 noninferiority trial. Fertil Steril. (2017) 107:387-96.e4. doi: $10.1016 /$ j.fertnstert.2016.10.033

75. D'Hooghe T, Longobardi S. Letter to Editor in Response to: Individualized Versus Conventional Ovarian Stimulation for in vitro Fertilization: A Multicenter, Randomized, Controlled, Assessor-Blinded, Phase 3 Noninferiority Trial. (2017). Available online at: https://www.fertstertdialog. com/users/16110-fertility-and-sterility/posts/12852--23086 (accessed December 21, 2018).

76. Wilkinson J, Lensen S. Letter to editor in response to: individualized versus conventional ovarian stimulation for in vitro fertilization: a multicenter, randomized, controlled, assessor-blinded, phase 3 noninferiority trial (2017) [cited 201926 Mar 2019]. Available online at: https://www.fertstertdialog. com/users/16110-fertility-and-sterility/posts/12852--23086.

77. Abd-Elaziz K, Duijkers I, Stöckl L, Dietrich B, Klipping C, Eckert K, et al. A new fully human recombinant FSH (follitropin epsilon): two phase I randomized placebo and comparator-controlled pharmacokinetic and pharmacodynamic trials. Hum Reprod. (2017) 32:1639-47. doi: 10.1093/humrep/dex220

78. Glycotope $\mathrm{GmbH}$, inventor; Glycotope $\mathrm{GmbH}$, assignee. US Patent for Recombinant Human Follicle-Stimulating Hormone Patent. Patent \# $9,527,899$. US (2011). 
79. Pouwer AW, Farquhar C, Kremer JA. Long-acting FSH versus daily FSH for women undergoing assisted reproduction. Cochrane Database Syst Rev. (2015) 2015:CD009577. doi: 10.1002/14651858.CD009577.pub3

80. Fares FA, Suganuma N, Nishimori K, LaPolt PS, Hsueh AJ, Boime I. Design of a long-acting follitropin agonist by fusing the C-terminal sequence of the chorionic gonadotropin beta subunit to the follitropin beta subunit. Proc Natl Acad Sci USA. (1992) 89:4304-8. doi: 10.1073/pnas.89.10.4304

81. Fauser BCJM, Alper MM, Ledger W, Schoolcraft WB, Zandvliet A, Mannaerts BMJL. Pharmacokinetics and follicular dynamics of corifollitropin alfa versus recombinant FSH during ovarian stimulation for IVF. Reprod Biomed Online. (2010) 21:593-601. doi: 10.1016/j.rbmo.2010.06.032

82. Ledger WL, Fauser BCJM, Devroey P, Zandvliet AS, Mannaerts BMJL. Corifollitropin alfa doses based on body weight: clinical overview of drug exposure and ovarian response. Reprod Biomed Online. (2011) 23:150-9. doi: 10.1016/j.rbmo.2011.04.002

83. Fensore S, Di Marzio M, Tiboni GM. Corifollitropin alfa compared to daily FSH in controlled ovarian stimulation for in vitro fertilization: a meta-analysis. J Ovarian Res. (2015) 8:33. doi: 10.1186/s13048-015-0160-4

84. Mahmoud Youssef MA, van Wely M, Aboulfoutouh I, El-Khyat W, van der Veen F, Al-Inany H. Is there a place for corifollitropin alfa in IVF/ICSI cycles? a systematic review and meta-analysis. Fertil Steril. (2012) 97:876-85. doi: 10.1016/j.fertnstert.2012.01.092

85. Low SC, Nunes SL, Bitonti AJ, Dumont JA. Oral and pulmonary delivery of $\mathrm{FSH}-\mathrm{Fc}$ fusion proteins via neonatal $\mathrm{Fc}$ receptor-mediated transcytosis. Hum Reprod. (2005) 20:1805-13. doi: 10.1093/humrep/deh896

86. Zhang Y-L, Guo K-P, Ji S-Y, Liu X-M, Wang P, Wu J, et al. Development and characterization of a novel long-acting recombinant follicle stimulating hormone agonist by fusing Fc to an FSH- $\beta$ subunit. Hum Reprod. (2016) 31:169-82. doi: 10.1093/humrep/dev295

87. Perlman S, van den Hazel B, Christiansen J, Gram-Nielsen S, Jeppesen CB, Andersen KV, et al. Glycosylation of an N-terminal extension prolongs the half-life and increases the in vivo activity of follicle stimulating hormone. $J$ Clin Endocrinol Metab. (2003) 88:3227-35. doi: 10.1210/jc.2002-021201

88. Klein J, Lobel L, Pollak S, Lustbader B, Ogden RT, Sauer MV, et al. Development and characterization of a long acting recombinant hFSH agonist. Hum Reprod. (2003) 18:50-6. doi: 10.1093/humrep/deg024

89. van Wely M, Kwan I, Burt AL, Thomas J, Vail A, Van der Veen F, et al. Recombinant versus urinary gonadotrophin for ovarian stimulation in assisted reproductive technology cycles. Cochrane Database Syst Rev. (2011) 2011:CD005354. doi: 10.1002/14651858.CD005354.pub2

90. Weiss NS, Kostova E, Nahuis M, Mol BWJ, van der Veen F, van Wely M. Gonadotrophins for ovulation induction in women with polycystic ovary syndrome. Cochrane Database Syst Rev. (2019) 1:CD010290. doi: 10.1002/14651858.CD010290.pub3

91. Lehert P, Schertz JC, Ezcurra D. Recombinant human follicle-stimulating hormone produces more oocytes with a lower total dose per cycle in assisted reproductive technologies compared with highly purified human menopausal gonadotrophin: a meta-analysis. Reprod Biol Endocrinol. (2010) 8:112. doi: 10.1186/1477-7827-8-112

92. Drakopoulos P, Errazuriz J, Santos-Ribeiro S, Tournaye H, Vaiarelli A, Pluchino N, et al. Cumulative live birth rates in IVF. Minerva Ginecol. (2018) 360:236-243. doi: 10.23736/S0026-4784.18.04347-2

93. Maheshwari A, McLernon D, Bhattacharya S. Cumulative live birth rate: time for a consensus? Hum Reprod. (2015) 30:2703-7. doi: 10.1093/humrep/dev263

94. Baker VL, Brown MB, Luke B, Conrad KP. Association of number of retrieved oocytes with live birth rate and birth weight: an analysis of 231,815 cycles of in vitro fertilization. Fertil Steril. (2015) 103:931-8 e2. doi: 10.1016/j.fertnstert.2014.12.120

95. Briggs R, Kovacs G, MacLachlan V, Motteram C, Baker HW. Can you ever collect too many oocytes? Hum Reprod. (2015) 30:81-7. doi: 10.1093/humrep/deu272

96. Steward RG, Lan L, Shah AA, Yeh JS, Price TM, Goldfarb JM, et al. Oocyte number as a predictor for ovarian hyperstimulation syndrome and live birth: an analysis of 256,381 in vitro fertilization cycles. Fertil Steril. (2014) 101:967-73. doi: 10.1016/j.fertnstert.2013.12.026
97. Sunkara SK, Rittenberg V, Raine-Fenning N, Bhattacharya S, Zamora J, Coomarasamy a. association between the number of eggs and live birth in IVF treatment: an analysis of 400135 treatment cycles. Hum Reprod. (2011) 26:1768-74. doi: 10.1093/humrep/der106

98. Magnusson A, Kallen K, Thurin-Kjellberg A, Bergh C. The number of oocytes retrieved during IVF: a balance between efficacy and safety. Hum Reprod. (2018) 33:58-64. doi: 10.1093/humrep/dex334

99. Malchau SS, Henningsen AA, Forman J, Loft A, Nyboe Andersen A, Pinborg A. Cumulative live birth rate prognosis based on the number of aspirated oocytes in previous ART cycles. Hum Reprod. (2019) 34:171-80. doi: 10.1093/humrep/dey341

100. Polyzos NP, Drakopoulos P, Parra J, Pellicer A, Santos-Ribeiro S, Tournaye $\mathrm{H}$, et al. Cumulative live birth rates according to the number of oocytes retrieved after the first ovarian stimulation for in vitro fertilization/intracytoplasmic sperm injection: a multicenter multinational analysis including approximately 15,000 women. Fertil Steril. (2018) 110:661-70 e1. doi: 10.1016/j.fertnstert.2018.04.039

101. Levi Setti PE, Alviggi C, Colombo GL, Pisanelli C, Ripellino C, Longobardi $S$, et al. Human recombinant follicle stimulating hormone (rFSH) compared to urinary human menopausal gonadotropin (HMG) for ovarian stimulation in assisted reproduction: a literature review and cost evaluation. J Endocrinol Invest. (2015) 38:497-503. doi: 10.1007/s40618-0140204-4

102. Bergh C, Howles CM, Borg K, Hamberger L, Josefsson B, Nilsson L, et al. Recombinant human follicle stimulating hormone (r-hFSH; Gonal-F) versus highly purified urinary FSH (Metrodin HP): results of a randomized comparative study in women undergoing assisted reproductive techniques. Hum Reprod. (1997) 12:2133-9. doi: 10.1093/humrep/12.10.2133

103. Frydman R, Howles CM, Truong F. A double-blind, randomized study to compare recombinant human follicle stimulating hormone (FSH; Gonal-F) with highly purified urinary FSH (Metrodin) HP) in women undergoing assisted reproductive techniques including intracytoplasmic sperm injection. The French Multicentre Trialists. Hum Reprod. (2000) 15:520-5. doi: 10.1093/humrep/15.3.520

104. Hedon B, Out HJ, Hugues JN, Camier B, Cohen J, Lopes P, et al. Efficacy and safety of recombinant follicle stimulating hormone (Puregon) in infertile women pituitary-suppressed with triptorelin undergoing in-vitro fertilization: a prospective, randomized, assessor-blind, multicentre trial. Hum Reprod. (1995) 10:3102-6. doi: 10.1093/oxfordjournals.humrep.a135866

105. Liu PY, Turner L, Rushford D, McDonald J, Baker HW, Conway AJ, et al. Efficacy and safety of recombinant human follicle stimulating hormone (Gonal-F) with urinary human chorionic gonadotrophin for induction of spermatogenesis and fertility in gonadotrophindeficient men. Hum Reprod. (1999) 14:1540-5. doi: 10.1093/humrep/14. 6.1540

106. Bouloux P, Warne DW, Loumaye E. Efficacy and safety of recombinant human follicle-stimulating hormone in men with isolated hypogonadotropic hypogonadism. Fertil Steril. (2002) 77:270-3. doi: 10.1016/S0015-0282(01)02973-9

107. Attia AM, Abou-Setta AM, Al-Inany HG. Gonadotrophins for idiopathic male factor subfertility. Cochrane Database Syst Rev. (2013) 2013:CD005071. doi: 10.1002/14651858.CD005071.pub4

108. Youssef MA, Abou-Setta AM, Lam WS. Recombinant versus urinary human chorionic gonadotrophin for final oocyte maturation triggering in IVF and ICSI cycles. Cochrane Database Syst Rev. (2016) 4:CD003719. doi: 10.1002/14651858.CD003719.pub4

109. European Medicines Agency. Ovitrelle. (2018). Available online at: https://www.ema.europa.eu/en/medicines/human/EPAR/ovitrelle (accessed January 30, 2019).

110. European Medicines Agency. Luveris. (2018). Available online at: https:// www.ema.europa.eu/en/medicines/human/EPAR/luveris (accessed January 30, 2019).

111. Trinchard-Lugan I, Khan A, Porchet HC, Munafo A. Pharmacokinetics and pharmacodynamics of recombinant human chorionic gonadotrophin in healthy male and female volunteers. Reprod Biomed Online. (2002) 4:106-15. doi: 10.1016/S1472-6483(10)61927-X 
112. Duffy DM, Hutchison JS, Stewart DR, Stouffer RL. Stimulation of primate luteal function by recombinant human chorionic gonadotropin and modulation of steroid, but not relaxin, production by an inhibitor of 3 beta-hydroxysteroid dehydrogenase during simulated early pregnancy. J Clin Endocrinol Metab. (1996) 81:2307-13. doi: 10.1210/jcem.81.6.8964869

113. Zelinski-Wooten MB, Hutchison JS, Trinchard-Lugan I, Hess DL, Wolf DP, Stouffer RL. Initiation of periovulatory events in gonadotrophin-stimulated macaques with varying doses of recombinant human chorionic gonadotrophin. Hum Reprod. (1997) 12:1877-85. doi: 10.1093/humrep/12.9.1877

114. Driscoll GL, Tyler JP, Hangan JT, Fisher PR, Birdsall MA, Knight DC. A prospective, randomized, controlled, double-blind, double-dummy comparison of recombinant and urinary HCG for inducing oocyte maturation and follicular luteinization in ovarian stimulation. Hum Reprod. (2000) 15:1305-10. doi: 10.1093/humrep/15.6.1305

115. International Recombinant Human Chorionic Gonadotropin Study Group. Induction of ovulation in World Health Organization group II anovulatory women undergoing follicular stimulation with recombinant human folliclestimulating hormone: a comparison of recombinant human chorionic gonadotropin (rhCG) and urinary hCG. Fertil Steril. (2001) 75:1111-8. doi: 10.1016/S0015-0282(01)01803-9

116. European Recombinant Human Chorionic Gonadotrophin Study Group. Induction of final follicular maturation and early luteinization in women undergoing ovulation induction for assisted reproduction treatmentrecombinant HCG versus urinary HCG. the european recombinant human chorionic gonadotrophin study group. Hum Reprod. (2000) 15:1446-51. doi: 10.1093/humrep/15.7.1446

117. Chang P, Kenley S, Burns T, Denton G, Currie K, DeVane G, et al. Recombinant human chorionic gonadotropin (rhCG) in assisted reproductive technology: results of a clinical trial comparing two doses of rhCG (Ovidrel) to urinary hCG (Profasi) for induction of final follicular maturation in in vitro fertilization-embryo transfer. Fertil Steril. (2001) 76:67-74. doi: 10.1016/S0015-0282(01)01851-9

118. Bellavia M, de Geyter C, Streuli I, Ibecheole V, Birkhauser MH, Cometti BP, et al. Randomized controlled trial comparing highly purified (HP-hCG) and recombinant hCG ( $\mathrm{r}$-hCG) for triggering ovulation in ART. Gynecol Endocrinol. (2013) 29:93-7. doi: 10.3109/09513590.2012. 730577

119. Shoham ZMD, Insler VMD. Recombinant technique and gonadotropins production: new era in reproductive medicine. Fertil Steril. (1998) 69:3S-15S. doi: 10.1016/S0015-0282(97)00506-2

120. le Cotonnec J-Y, Porchet H, Beltrami V, Munafo A. Clinical pharmacology of recombinant human luteinizing hormone: part I. pharmacokinetics after intravenous administration to healthy female volunteers and comparison with urinary human luteinizing hormone. Fertil Steril. (1998) 69:189-94. doi: 10.1016/S0015-0282(97)00501-3

121. Merck Serono. Luveris Summary of Product Characteristics. (2018). Available online at: https://www.medicines.org.uk/emc/product/1573/smpc (accessed July 24, 2019).

122. Merck. Pergoveris, Russian Summary of Product Characteristics. (2017).

123. European Medicines Agency. Pergoveris. (2018). Available online at: https:// www.ema.europa.eu/en/medicines/human/EPAR/pergoveris (accessed January 30, 2019).

124. Dhillon S, Keating GM. Lutropin Alfa. Drugs. (2008) 68:1529-40. doi: 10.2165/00003495-200868110-00005

125. The European Recombinant Human LH Study Group. Recombinant human luteinizing hormone (LH) to support recombinant human folliclestimulating hormone (FSH)-induced follicular development in $\mathrm{LH}$ - and FSH-deficient anovulatory women: a dose-finding study. J Clin Endocrinol Metab. (1998) 83:1507-14. doi: 10.1210/jc.83.5.1507

126. Burgues $\mathrm{S}$. The effectiveness and safety of recombinant human LH to support follicular development induced by recombinant human FSH in WHO group I anovulation: evidence from a multicentre study in Spain. Hum Reprod. (2001) 16:2525-32. doi: 10.1093/humrep/16.12.2525

127. De Moustier B, Brinsden P, Bungum L, Fisch B, Pinkstone S, Warne $\mathrm{D}$, et al. 0-158. The effects of combined treatment of recombinant (r)FSH and rLH in ratios 1:1, 2:1 and 3:1 in women. aged 38-42 years undergoing IVF-ICSI treatment. Hum Reprod. (2002) 17(Suppl 1):55. doi: 10.1093/humrep/17.suppl_1.54

128. Humaidan P, Chin W, Rogoff D, D'Hooghe T, Longobardi S, Hubbard J, et al. Efficacy and safety of follitropin alfa/lutropin alfa in ART: a randomized controlled trial in poor ovarian responders. Hum Reprod. (2017) 32:1537-8. doi: 10.1093/humrep/dex208

129. Humaidan P, Chin W, Rogoff D, D'Hooghe T, Longobardi S, Hubbard J, et al. Efficacy and safety of follitropin alfa/lutropin alfa in ART: a randomized controlled trial in poor ovarian responders. Hum Reprod. (2017) 32:544-55. doi: 10.1093/humrep/dew360

130. Lehert P, Chin W, Schertz J, D'Hooghe T, Alviggi C, Humaidan P. Predicting live birth for poor ovarian responders: the PROsPeR concept. Reprod Biomed Online. (2018) 37:43-52. doi: 10.1016/j.rbmo.2018.03.013

131. Alviggi C, Conforti A, Esteves SC, Andersen CY, Bosch E, Buhler $\mathrm{K}$, et al. Recombinant luteinizing hormone supplementation in assisted reproductive technology: a systematic review. Fertil Steril. (2018) 109:64464. doi: 10.1016/j.fertnstert.2018.01.003

132. Conforti A, Esteves SC, Di Rella F, Strina I, De Rosa P, Fiorenza A, et al. The role of recombinant $\mathrm{LH}$ in women with hypo-response to controlled ovarian stimulation: a systematic review and meta-analysis. Reprod Biol Endocrinol. (2019) 17:18. doi: 10.1186/s12958-019-0460-4

133. Lehert P, Kolibianakis EM, Venetis CA, Schertz J, Saunders H, Arriagada $\mathrm{P}$, et al. Recombinant human follicle-stimulating hormone (r-hFSH) plus recombinant luteinizing hormone versus r-hFSH alone for ovarian stimulation during assisted reproductive technology: systematic review and meta-analysis. Reprod Biol Endocrinol. (2014) 12:17. doi: 10.1186/1477-7827-12-17

134. Mochtar MH, Danhof NA, Ayeleke RO, Van der Veen F, van Wely M. Recombinant luteinizing hormone (rLH) and recombinant follicle stimulating hormone $(\mathrm{rFSH})$ for ovarian stimulation in IVF/ICSI cycles. Cochrane Database Syst Rev. (2017) 5:CD005070. doi: 10.1002/14651858.CD005070.pub3

135. Santi D, Casarini L, Alviggi C, Simoni M. Efficacy of folliclestimulating hormone (FSH) alone, FSH + luteinizing hormone, human menopausal gonadotropin or FSH + human chorionic gonadotropin on assisted reproductive technology outcomes in the "personalized" medicine era: a meta-analysis. Front Endocrinol. (2017) 8:114. doi: 10.3389/fendo.2017.00114

136. Braakhekke M, Kamphuis EI, Dancet EA, Mol F, van der Veen F, Mol BW. Ongoing pregnancy qualifies best as the primary outcome measure of choice in trials in reproductive medicine: an opinion paper. Fertil Steril. (2014) 101:1203-4. doi: 10.1016/j.fertnstert.2014.03.047

137. Clarke JF, van Rumste MM, Farquhar CM, Johnson NP, Mol BW, Herbison P. Measuring outcomes in fertility trials: can we rely on clinical pregnancy rates? Fertil Steril. (2010) 94:1647-51. doi: 10.1016/j.fertnstert.2009.11.018

138. Martins WP, Niederberger C, Nastri CO, Racowsky C. Making evidencebased decisions in reproductive medicine. Fertil Steril. (2018) 110:1227-30. doi: 10.1016/j.fertnstert.2018.08.010

139. Mol BW, Bossuyt PM, Sunkara SK, Garcia Velasco JA, Venetis C, Sakkas D, et al. Personalized ovarian stimulation for ART: study design considerations to move from hype to added value for patients. Fertil Steril. (2018) 109:968-79. doi: 10.1016/j.fertnstert.2018. 04.037

140. Alviggi C, Conforti A, Esteves SC, Vallone R, Venturella R, Staiano S, et al. Understanding ovarian hypo-response to exogenous gonadotropin in ovarian stimulation and its new proposed marker-the follicle-to-oocyte (FOI) index. Front Endocrinol. (2018) 9:589. doi: 10.3389/fendo.2018. 00589

141. Gerrits MG, Kramer H, el Galta R, van Beerendonk G, Hanssen R, AbdElaziz K, et al. Oral follicle-stimulating hormone agonist tested in healthy young women of reproductive age failed to demonstrate effect on follicular development but affected thyroid function. Fertil Steril. (2016) 105:1056-62 e4. doi: 10.1016/j.fertnstert.2015.12.017

142. Abbotts C, Salgado-Braga C, Audibert-Gros C. A redesigned follitropin alfa pen injector for infertility: results of a market research study. Patient Prefer Adherence. (2011) 5:315-31. doi: 10.2147/PPA. S21421 
143. Schertz J, Worton H. Nurse evaluation of the redesigned fertility pen injector: a questionnaire-based observational survey. Expert Opin Drug Deliv. (2018) 15:435-42. doi: 10.1080/17425247.2018.1450386

144. Schertz J, Worton H. Patient evaluation of the redesigned follitropin alfa pen injector. Expert Opin Drug Deliv. (2017) 14:473-81. doi: $10.1080 / 17425247.2017 .1289174$

Conflict of Interest Statement: WB is an employee of Merck Serono GmbH, Darmstadt, Germany. SL and TD are employees of Merck Healthcare KGaA, Darmstadt, Germany. VA is an employee of EMD Serono, Rockland, MA, USA, a business of Merck KGaA, Darmstadt, Germany. SS has received grants and nonfinancial support from Merck and Ferring.
The remaining author declares that the research was conducted in the absence of any commercial or financial relationships that could be construed as a potential conflict of interest.

Copyright (c) 2019 Lunenfeld, Bilger, Longobardi, Alam, D'Hooghe and Sunkara. This is an open-access article distributed under the terms of the Creative Commons Attribution License (CC BY). The use, distribution or reproduction in other forums is permitted, provided the original author(s) and the copyright owner(s) are credited and that the original publication in this journal is cited, in accordance with accepted academic practice. No use, distribution or reproduction is permitted which does not comply with these terms. 